\title{
Comparison of KRAS/BRAF mutations between primary tumors and serum in colorectal cancer: Biological and clinical implications
}

\author{
XINGXIANG PU ${ }^{1-3^{*}}$, ZHIZHONG PAN ${ }^{3,4^{*}}$, YING HUANG ${ }^{3,5^{*}}$, YING TIAN $^{3,6}$, HONGQIANG GUO ${ }^{1,3}$,

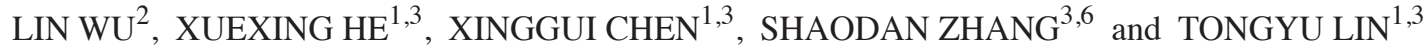 \\ ${ }^{1}$ Department of Medical Oncology, Cancer Center, Sun Yat-sen University, Guangzhou 510060; \\ ${ }^{2}$ Department of Medical Oncology, Hu Nan Provincal Tumor Hospital, ChangSha, 410013; \\ ${ }^{3}$ State Key Laboratory of Oncology in Southern China; Departments of ${ }^{4}$ Colorectal Surgery and ${ }^{5}$ Radiotherapy; \\ ${ }^{6}$ Research Department, Cancer Center, Sun Yat-sen University, Guangzhou 510060, P.R. China
}

Received March 5, 2012; Accepted June 14, 2012

DOI: $10.3892 / 01.2012 .963$

\begin{abstract}
In colorectal cancer (CRC), KRAS and BRAF mutations in primary tumors are associated with resistance to anti-epidermal growth factor receptor (anti-EGFR)-based therapies. However, the correlation between KRAS/BRAF mutation in primary tumors and serum has not been well studied. To evaluate the degree of concordance of KRAS/BRAF mutations between the primary tumors and the matched serum samples in CRC, serum and tumor tissues were collected from 115 patients with CRC and KRAS/BRAF mutations were examined by nested polymerase chain reaction (PCR) and direct sequencing. BRAF mutations were present in $3.5 \%(4 / 115)$ of the primary tumor tissue samples and $0.87 \%$ $(1 / 115)$ of the serum samples. In the 4 primary tumors with BRAF mutations, identical mutations were not observed in the corresponding serum samples $(\kappa=-0.016)$. KRAS mutations were observed in $32.2 \%(37 / 115)$ of the primary tumors and $11.3 \%(13 / 115)$ of the serum samples. Of the 37 tumor cases with KRAS mutations, 9 had identical mutations in the corresponding serum sample, with a concordance rate of $24.3 \%$ (9/37). Discordance was observed in $32(27.8 \%)$ patients. The concordance between KRAS mutations in the primary tumors and KRAS mutations in the matched serums was low $(\kappa=0.231)$. The results of the present study suggest that the possibility of differences in the mutational status of KRAS/BRAF between primary tumors and matched serum samples should be considered when patients are selected for anti-EGFR-based therapies.
\end{abstract}

Correspondence to: Dr Tongyu Lin, Department of Medical Oncology, Cancer Center, Sun Yat-sen University, 651 DongFeng Road, Guangzhou 510060, P.R. China

E-mail: tongyulin@hotmail.com

*Contributed equally

Key words: colorectal cancer, cetuximab, KRAS, BRAF, serum

\section{Introduction}

Colorectal cancer (CRC) is one of the leading causes of mortality in the world. It was estimated that over 145,000 patients were diagnosed with CRC and nearly 50,000 individuals succumbed to the disease in the USA in 2009 (1). In China, the incidence of CRC is increasing each year. CRC has become the second most common type of cancer in certain regions, such as Shanghai.

Approximately $60 \%$ of patients newly diagnosed with CRC in China have an advanced stage of the disease and 50-60\% of CRC patients develop metastatic CRC (mCRC) $(2,3)$. Chemotherapy prolongs the survival of patients with advanced CRC and high risk stage II patients $(4,5)$. The development of cetuximab/panitumumab (monoclonal antibodies directed against the epidermal growth factor receptor) holds promise for patients with CRC (6). It has been verified that chemotherapy combined with cetuximab/panitumumab improves the overall and progression-free survival rates, compared with chemotherapy alone, but the benefits of cetuximab/panitumumab are limited to the patients with wild-type KRAS (6-9). KRAS mutation is an independent prognostic factor in patients with $\mathrm{mCRC}$ treated with cetuximab/panitumumab (9-11).

The occurrence of KRAS mutations accounts for $30-40 \%$ of patients who are nonresponsive to anti-EGFR therapy (12-14). This suggests that there are other molecular factors determining the responses to cetuximab treatment. It has been reported that various factors may be associated with response to cetuximab treatment in CRC, including NF- $\mathrm{kb}$, PI3KCA, PTEN and BRAF (15-17). Mutation of the BRAF gene is one of factors that cause resistance to cetuximab in patients with the wild-type KRAS gene $(18,19)$. Detection of KRAS gene mutation prior to the use of cetuximab in CRC therapy has been recommended by NCCN guidelines. BRAF gene mutation detection is also necessary before the administration of cetuximab therapy. However, for certain patients, it is difficult to obtain tumor samples suitable for the analysis of KRAS/BRAF mutations. Another problem is that cetuximab is currently used mostly for patients with mCRC. Considering 
the genetic heterogeneity of CRC, the absence of detectable KRAS/BRAF mutation in the primary tumor cannot exclude the presence of a KRAS/BRAF mutation in the metastases (20). In addition, it is difficult to obtain metastatic tumor tissues for analysis of KRAS/BRAF mutations. It is necessary to identify alternative methods to detect KRAS/BRAF gene mutations in CRC patients treated with anti-EGFR.

The detection of KRAS/BRAF mutations in serum or plasma is one of the alternatives and may have clinical relevance before conducting anti-EGFR therapies in patients with CRC. The correlation between KRAS/BRAF mutations in primary tumors and serum has not been well studied. The present study was conducted to investigate the degree of concordance between the KRAS/BRAF mutations in colorectal tumor tissues and those in the matched serum samples. We aim to identify whether the detection of KRAS/BRAF in serum predicts mutations of these genes in the primary tumor tissues of CRC.

\section{Materials and methods}

Patients. Between February 2002 and February 2010, a total of 115 patients were enrolled from Sun Yat-Sen University Cancer Center (Guangzhou, China). Colorectal adenocarcinoma in these patients was confirmed by histological examination. The stage of the disease corresponds to that at the time of primary diagnosis, and was determined by abdominal computed tomography or operative findings according to the American Joint Committee on Cancer (AJCC) TNM staging system for CRC (2002). Informed consent was obtained from all participants for the use of their tumor and blood samples in this study. This project was approved by the Clinical Research Ethics Committee of Sun Yat-sen University Cancer Center, Guangzhou, China.

Sample preparation and DNA extraction. Representative samples of tumor tissues were obtained immediately following surgical resection. The tissue samples were stored at $-80^{\circ} \mathrm{C}$ after immediate freezing in liquid nitrogen. Prior to DNA extraction, a fragment of each sample was fixed in formalin to confirm the presence of neoplastic tissue by histopathological analysis. Peripheral blood $(5 \mathrm{ml})$ samples were collected immediately prior to surgical resection into EDTA anticoagulative tubes. Blood samples were centrifuged for $15 \mathrm{~min}$ at $1,500 \mathrm{rpm}$ to separate buffy coats and plasma. DNA was extracted from the serum $(200 \mu \mathrm{l})$ and tumor tissue $(25 \mathrm{mg})$ samples by QIAmp Blood and Tissue kit (Qiagen, Hilden, Germany) and was eluted in a final volume of 30 and $50 \mu 1$, respectively.

Detection of KRAS/BRAF mutations. Mutation analyses of KRAS at codons 12 and 13 and BRAF at codon 600 were performed by nested polymerase chain reaction (PCR). The following nested primers were used: KRAS: F, 5'-GTGTGA CATGTTCTAATATAGTCA-3'; R, 5'-GAATGGTCCTGC ACCAGTAA-3'; Fint, 5'-ATGTTCTAATATAGTCACATT TTC-3'; and Rint, 5'-GTCCTGCACCAGTAATATGC-3'. BRAF: F, 5'-TCATAATGCTTGCTCTGATAGGA-3'; R, 5'-GGCCAAAAATTTAATCAGTGGA-3'; Fint, 5'-GCTTGC TCTGATAGGAAAATG-3'; Rint, 5'-ATAGCCTCAATTCTT ACCATCC-3'.
Table I. Characteristics of patients included in this study.

\begin{tabular}{lcc}
\hline Characteristics & No. & Percentage \\
\hline Patients & 115 & \\
Age (years) & & \\
Median & 58 & \\
Range & $24-89$ & \\
Gender & & \\
Female & 39 & \\
Male & 76 & \\
Tumor stage & & 6.1 \\
I & 7 & 33.0 \\
II (A/B) & 38 & 34.8 \\
III (A/B/C) & 40 & 26.1 \\
IV & 30 & \\
Site of primary disease & & 60.0 \\
Colon & 69 & 40.0 \\
Rectum & 46 & \\
\hline
\end{tabular}

Each 50- $\mu 1$ PCR cocktail contained $3 \mu 1$ genomic DNA, $3.0 \mathrm{mM} \mathrm{Mg}{ }^{2+}, 0.4 \mathrm{mM}$ dNTP and 1.5 units Taq polymerase (Promega, Madison, WI, USA). PCR parameters for detection of the KRAS gene were 1 cycle at $94^{\circ} \mathrm{C}$ for 5 min followed by 30 cycles of $94^{\circ} \mathrm{C}$ for $30 \mathrm{sec}, 54^{\circ} \mathrm{C}$ for $30 \mathrm{sec}$ and $72^{\circ} \mathrm{C}$ for $40 \mathrm{sec}$ and a final cycle of $72^{\circ} \mathrm{C}$ for $5 \mathrm{~min}$. PCR parameters for detection of the BRAF gene were 1 cycle of $94^{\circ} \mathrm{C}$ for $5 \mathrm{~min}$ followed by 35 cycles of $94^{\circ} \mathrm{C}$ for $30 \mathrm{sec}, 55^{\circ} \mathrm{C}$ for $30 \mathrm{sec}, 72^{\circ} \mathrm{C}$ for $40 \mathrm{sec}$ and a final cycle of $72^{\circ} \mathrm{C}$ for $5 \mathrm{~min}$. PCR products were quantified by running on a $2 \%$ agarose gel.

Sequence analysis was performed by Shanghai Invitrogen Biotechnology Co., Ltd. Guangzhou Office (China). The sequences were analyzed by Chromas and visual inspection of each sequence in forward and reverse directions.

Statistical analysis. Statistical analysis was performed using the SPSS 13.0 software for Windows (SPSS, Inc., Chicago, IL, USA). Chi-square and $\kappa$ coefficient tests were used to compare the occurrence and the degree of concordance of KRAS/BRAF mutation between tumor and serum samples.

\section{Results}

Patient characteristics. Of the 115 patients, 76 (66.1\%) were male and 39 (33.9\%) were female. Their median age was 58 years (range, 24-89). A total of $69(60 \%)$ of the primary tumors of the patients were located in the colon and the remaining $46(40 \%)$ were located in the rectum. The tumor stage distribution was as follows: $6.1 \%(7 / 115)$ of the patients were stage I, $33.0 \%$ (38/115) were stage II, 34.8\% (40/115) were stage III and $26.1 \%$ (30/115) were stage IV. The clinicopathological characteristics of the patients are presented in Table I.

KRAS mutation status of the primary tumours and the corresponding serum samples. Among the 115 matched serum and tumor tissue samples, KRAS mutations were observed 
Table II. KRAS/BRAF mutations in the primary tumor tissues and their matched serum samples.

\begin{tabular}{|c|c|c|c|c|}
\hline \multirow{2}{*}{$\begin{array}{l}\text { Case } \\
\text { no. }\end{array}$} & \multicolumn{2}{|c|}{ KRAS } & \multicolumn{2}{|c|}{ BRAF } \\
\hline & Primary tumor & Serum & Primary tumor & Serum \\
\hline 1 & $\mathrm{~m}(\mathrm{G} 12 \mathrm{D})$ & w & w & w \\
\hline 2 & $\mathrm{~m}(\mathrm{G} 12 \mathrm{D})$ & $\mathrm{w}$ & w & w \\
\hline 3 & $\mathrm{~m}(\mathrm{G} 12 \mathrm{D})$ & w & w & w \\
\hline 4 & $\mathrm{~m}(\mathrm{G} 12 \mathrm{D})$ & w & W & w \\
\hline 5 & $\mathrm{~m}(\mathrm{G} 12 \mathrm{D})$ & $\mathrm{w}$ & $\mathrm{w}$ & w \\
\hline 6 & $\mathrm{~m}(\mathrm{G} 12 \mathrm{D})$ & $\mathrm{w}$ & w & w \\
\hline 7 & $\mathrm{~m}(\mathrm{G} 12 \mathrm{D})$ & $\mathrm{w}$ & $\mathrm{w}$ & w \\
\hline 8 & $\mathrm{~m}(\mathrm{G} 12 \mathrm{D})$ & w & $\mathrm{w}$ & w \\
\hline 9 & $\mathrm{~m}(\mathrm{G} 12 \mathrm{D})$ & $\mathrm{w}$ & w & w \\
\hline 10 & $\mathrm{~m}(\mathrm{G} 12 \mathrm{D})$ & $\mathrm{w}$ & w & w \\
\hline 11 & $\mathrm{~m}(\mathrm{G} 12 \mathrm{D})$ & $\mathrm{w}$ & w & w \\
\hline 12 & $\mathrm{~m}(\mathrm{G} 12 \mathrm{D})$ & $\mathrm{m}(\mathrm{G} 13 \mathrm{D})$ & w & w \\
\hline 13 & $\mathrm{~m}(\mathrm{G} 12 \mathrm{D})$ & $\mathrm{w}$ & w & w \\
\hline 14 & $\mathrm{~m}(\mathrm{G} 12 \mathrm{D})$ & $\mathrm{w}$ & w & w \\
\hline 15 & $\mathrm{~m}(\mathrm{G} 12 \mathrm{D})$ & $\mathrm{m}(\mathrm{G} 12 \mathrm{D})$ & w & w \\
\hline 16 & $\mathrm{~m}(\mathrm{G} 12 \mathrm{~V})$ & $\mathrm{w}$ & w & w \\
\hline 17 & $\mathrm{~m}(\mathrm{G} 12 \mathrm{~V})$ & w & w & w \\
\hline 18 & $\mathrm{~m}(\mathrm{G} 12 \mathrm{~V})$ & $\mathrm{w}$ & w & w \\
\hline 19 & $\mathrm{~m}(\mathrm{G} 12 \mathrm{~V})$ & $\mathrm{w}$ & w & w \\
\hline 20 & $\mathrm{~m}(\mathrm{G} 12 \mathrm{~V})$ & $\mathrm{m}(\mathrm{G} 12 \mathrm{~V})$ & w & w \\
\hline 21 & $\mathrm{~m}(\mathrm{G} 12 \mathrm{~V})$ & w & w & w \\
\hline 22 & $\mathrm{~m}(\mathrm{G} 13 \mathrm{R})$ & w & w & w \\
\hline 23 & $\mathrm{~m}(\mathrm{G} 13 \mathrm{D})$ & w & w & w \\
\hline 24 & $\mathrm{~m}(\mathrm{G} 13 \mathrm{D})$ & w & w & w \\
\hline 25 & $\mathrm{~m}(\mathrm{G} 13 \mathrm{D})$ & w & w & w \\
\hline 26 & $\mathrm{~m}(\mathrm{G} 13 \mathrm{D})$ & w & w & w \\
\hline 27 & $\mathrm{~m}(\mathrm{G} 13 \mathrm{D})$ & w & w & w \\
\hline 28 & $\mathrm{~m}(\mathrm{G} 13 \mathrm{D})$ & $\mathrm{m}(\mathrm{G} 12 \mathrm{~V})$ & w & w \\
\hline 29 & $\mathrm{~m}(\mathrm{G} 13 \mathrm{D})$ & $\mathrm{m}(\mathrm{G} 12 \mathrm{~V})$ & $\mathrm{w}$ & $\mathrm{w}$ \\
\hline 30 & $\mathrm{~m}(\mathrm{G} 13 \mathrm{D})$ & $\mathrm{w}$ & w & $\mathrm{w}$ \\
\hline 31 & $\mathrm{~m}(\mathrm{G} 13 \mathrm{D})$ & $\mathrm{m}(\mathrm{G} 12 \mathrm{D})$ & w & w \\
\hline 32 & $\mathrm{~m}(\mathrm{G} 13 \mathrm{D})$ & $\mathrm{m}(\mathrm{G} 12 \mathrm{~V})$ & w & w \\
\hline 33 & $\mathrm{~m}(\mathrm{G} 13 \mathrm{D})$ & $\mathrm{m}(\mathrm{G} 12 \mathrm{~V})$ & w & w \\
\hline 34 & $\mathrm{~m}(\mathrm{G} 13 \mathrm{D})$ & w & w & w \\
\hline 35 & w & $\mathrm{m}(\mathrm{G} 12 \mathrm{D})$ & w & w \\
\hline 36 & w & $\mathrm{m}(\mathrm{G} 12 \mathrm{~V})$ & w & w \\
\hline 37 & w & $\mathrm{m}(\mathrm{G} 12 \mathrm{~V})$ & w & $\mathrm{w}$ \\
\hline 38 & w & $\mathrm{m}(\mathrm{G} 12 \mathrm{D})$ & W & w \\
\hline 39 & w & w & m(V600E) & $\mathrm{w}$ \\
\hline 40 & w & w & $\mathrm{m}(\mathrm{V} 600 \mathrm{E})$ & w \\
\hline 41 & w & w & $\mathrm{m}(\mathrm{V} 600 \mathrm{E})$ & w \\
\hline 42 & w & w & $\mathrm{m}(\mathrm{V} 600 \mathrm{E})$ & W \\
\hline 43 & w & w & w & $\mathrm{m}(\mathrm{V} 600 \mathrm{E})$ \\
\hline 44 & $\mathrm{~m}(\mathrm{G} 12 \mathrm{~S})$ & $\mathrm{m}(\mathrm{G} 12 \mathrm{~S})$ & $\mathrm{w}$ & w \\
\hline 45 & $\mathrm{~m}(\mathrm{G} 12 \mathrm{~V})$ & w & w & w \\
\hline 46 & $\mathrm{~m}(\mathrm{G} 12 \mathrm{D})$ & w & $\mathrm{W}$ & w \\
\hline
\end{tabular}

w, wild-type; m, mutation.
Table III. Correlation between KRAS mutation in serum and primary tumor tissues.

\begin{tabular}{llr}
\hline & \multicolumn{2}{c}{ KRAS in serum } \\
\cline { 2 - 3 } KRAS in primary tumor & W & M \\
\hline W & 74 & 4 \\
M & 28 & 9
\end{tabular}

$\chi^{2}=7.408, P=0.006 ; \kappa=0.231(\kappa \geq 0.7$ indicates high degree of coincidence; $0.7>\kappa \geq 0.4$ indicates a middle degree of coincidence; $\kappa<0.4$ indicates low degree of coincidence). W, wild-type; $\mathrm{M}$, mutation.

Table IV. The correlation between KRAS mutation in serum and stage I and II primary tumor tissues.

\begin{tabular}{lcr}
\hline & \multicolumn{2}{c}{ KRAS in serum } \\
\cline { 2 - 3 } KRAS in primary tumor & W & M \\
\hline W & 28 & 1 \\
M & 13 & 3 \\
\hline P=0.002, $\kappa=0.184$. W, wild-type; M, mutation. & \\
\hline
\end{tabular}

in $32.2 \%(37 / 115)$ of the primary tumor tissue samples and $11.3 \%(13 / 115)$ of the serum samples. Of the 37 patients with KRAS mutations in the tumor tissue samples, 9 had an identical mutation in the serum sample, with a concordance rate of $24.3 \%$ (9/37). However, only 3 of the 9 identical KRAS mutations were located in the same codon. The remaining 6 identical KRAS mutations were located in different codons. A detailed codon distribution of specific KRAS mutations is shown in Table II. Discordance of the KRAS mutation was observed in $32(27.8 \%)$ patients, including 28 patients with a KRAS mutation in tumor tissues but wild-type KRAS in the serum and 4 patients with KRAS mutation in the serum but wild-type KRAS in tumor tissues (Table II). The KRAS mutation rate in serum samples was significantly different from that in the primary tumor tissue samples in CRC $\left(\chi^{2}=7.408, P=0.006\right)$. The concordance of the KRAS mutation rate between tumor tissue and blood samples was low ( $\kappa=0.231$; Table III).

The concordance of KRAS mutation between the primary tumor tissue and the corresponding serum samples was also analyzed according to the tumor stage. Low concordance of KRAS mutation between the primary tumors and the corresponding serum samples was observed regardless of tumor stage (Tables IV, V and VI).

BRAF mutation status of the primary tumours and the corresponding serum samples. BRAF mutations were present in 4 tumor tissues, accounting for $3.5 \%$ (4/115) of the primary tumor tissues, and only 1 serum sample, accounting for $0.87 \%$ $(1 / 115)$ of the serum samples. There were no identical BRAF mutations in serum samples in all the 4 patients with BRAF mutations in the primary tumors. The concordance of the 
Table V. Correlation between KRAS mutation in serum and stage III primary tumor tissues.

\begin{tabular}{lrr}
\hline & \multicolumn{2}{c}{ KRAS in serum } \\
\cline { 2 - 3 } KRAS in primary tumor & W & M \\
\hline W & 29 & 2 \\
M & 7 & 2 \\
\hline
\end{tabular}

$\mathrm{P}=0.180, \kappa=0.196 . \mathrm{W}$, wild-type; $\mathrm{M}$, mutation.

Table VI. Correlation between KRAS mutation in serum and stage IV primary tumor tissues.

\begin{tabular}{lrr}
\hline & \multicolumn{2}{c}{ KRAS in serum } \\
\cline { 2 - 3 } KRAS in primary tumor & $\mathrm{W}$ & $\mathrm{M}$ \\
\hline $\mathrm{W}$ & 17 & 1 \\
$\mathrm{M}$ & 8 & 4 \\
\hline
\end{tabular}

$\mathrm{P}=0.039, \kappa=0.308 . \mathrm{W}$, wild-type; $\mathrm{M}$, mutation.

Table VII. Correlation between BRAF mutation in serum and primary tumor tissues.

\begin{tabular}{lrr}
\hline & \multicolumn{2}{c}{ BRAF in serum } \\
\cline { 2 - 3 } BRAF in primary tumor & W & M \\
\hline W & 110 & 1 \\
M & 4 & 0
\end{tabular}

$\kappa=-0.014(\kappa \geq 0.7$ indicates high degree of coincidence; $0.7>\kappa \geq 0.4$ indicates middle degree of coincidence; $\kappa<0.4$ indicates low degree of coincidence). W, wild-type; $\mathrm{M}$, mutation.

BRAF mutation rate between tumor tissue and blood samples was low ( $\kappa=-0.014$; Tables II and VII).

\section{Discussion}

KRAS and BRAF are members of the MAP kinase (MAPK) pathway, which is hyperactivated in approximately $30 \%$ of all cancers (21). KRAS and BRAF mutations are associated with carcinogenesis and progression and resistance to anti-EGFR-based therapies in CRC (22). In the present study, KRAS mutation were found in $32.2 \%$ of Chinese CRC patients and 5 types of common KRAS mutation were observed. This is similar to the results of previous studies, which found that KRAS mutation occurs in 27 to $50 \%$ of CRC patients in western countries $(9,11,23)$. It has been reported that BRAF and KRAS mutations are mutually exclusive $(24,25)$. In this study, BRAF mutation was found only in patients with wild-type KRAS. The mutation rate of BRAF was $3.5 \%$ in primary tumor tissues, which is lower than that in previous studies; approximately $10 \%$ in CRC $(19,22,26)$. This result indicates that Chinese CRC patients with the wild-type KRAS gene may gain greater benefit from cetuximab therapy. There was no significant correlation between tumor stage and KRAS mutation in serum or primary tumor tissue samples, which was similar to previous studies $(27,28)$.

Previous studies have revealed that apoptosis or necrosis (or both) of tumor cells are the main source of free circulating DNA in serum. Another plausible mechanism was that free circulating DNA was actively released by living cells (29). Therefore, it is possible that the detection of KRAS/BRAF gene mutations in serum reflects their status in the primary tumor tissues. If there is high degree of concordance between the KRAS/BRAF mutation in serum and primary tumor tissues, the detection of KRAS/BRAF mutation in tumor tissues may be substituted by the detection of gene mutations in serum samples, since obtaining peripheral blood samples is noninvasive and convenient in comparison to tumor tissue samples.

In the present study, we first evaluated the degree of concordance between KRAS and BRAF mutations in serum samples and those in the primary tumor tissues in cases of CRC. Notably, no identical BRAF mutations were found between the serum samples and the matched primary tumors. The rate of KRAS mutation was $11.3 \%$ (13/115) in serum samples. The concordance of KRAS mutation rate between tumor tissue and blood samples was low.

Our results were similar to those of the study by Tórtola et al, which found that KRAS mutations were found in 17 primary tumor tissues and 6 bone marrow samples. Of the 17 KRAS mutations in the primary tumor tissues, 15 were located in codon 12 and 5 of the 6 mutations in bone marrow samples were located in codon 13 (30). We found that only 3 of the 9 identical KRAS mutations were located in the same codon, while the remaining 6 identical KRAS mutations were located in different codons. Albanese et al also reported a discordance between KRAS mutations in primary colorectal carcinomas and matched liver metastases (31). Another study on CRC revealed that the frequency of KRAS mutations in metastatic lymph nodes was higher than that in the primary tumors (22). Discordance of KRAS mutation was observed in 9 patients, including 2 patients with KRAS mutation in the primary tumor tissue but wild-type KRAS in metastatic lymph nude and 7 patients with KRAS mutation in metastatic lymph nodes but wild-type KRAS in the primary tumor tissues (22). Similar phenomena were also observed in non-small cell lung cancers (NSCLC). Discordance of EGFR mutations between primary tumors and the matched metastatic tumors or the corresponding serum samples have been reported in several studies (32-34). These results indicate that the analysis of EGFR mutations in the primary lung tumor tissues would be inadequate for the utilization of tyrosine kinase inhibitors (TKIs) for advanced NSCLC.

In our study, patients with stage I to IV disease were included. KRAS mutation may be detected in CRC patients ranging from early (dysplastic adenoma) to mCRC (35). Yen et al reported that KRAS status in the primary colorectal tumors was highly concordant with that in circulating tumor cells (CTCs) in mCRC patients (36). We also analyzed the 
concordance of KRAS mutation between the primary tumor tissue and the corresponding serum samples according to the tumor stage. The concordance of KRAS mutation was higher in cases of $\mathrm{mCRC}$ than in patients with early stage disease. We failed to find a high degree of coincidence of KRAS mutation even in mCRC. This may partially be explained by the insignificant increase in the prevalence of serum mutant KRAS positivity with advancing disease stage (35)

The results of the current study and previous studies indicate that circulating DNA or disseminated tumor cells are not always clonal with the primary tumors and a certain level of heterogeneity exists (29). Another possibility is that a KRAS/BRAF mutation may be acquired during the process of metastasis (24). The tumor tissues that carried mutations shed less DNA into the plasma than the other parts of the tumor tissues, in which case the detection of such mutations in the plasma may be missed. In addition, the released DNA in the plasma is diluted, which impedes the detection of mutations in plasma DNA despite of the presence of mutations in tumors (32). The discordant KRAS/BRAF mutations may partially account for the fact that certain CRC patients with wild-type KRAS/BRAF in primary tumors fail to respond to cetuximab or panitumumab therapies. These results also suggest that the analysis of KRAS/BRAF mutation in the primary tumor tissues would not be adequate before performing cetuximab and panitumumab therapies in patients with CRC.

In conclusion, we have demonstrated that circulating KRAS/BRAF mutations in serum are not always consistent with those in the primary colorectal tumors. The resistance of certain CRC patients with wild-type KRAS/BRAF to cetuximab- or panitumumab-based therapies may be partially caused by the discordant KRAS/BRAF mutations. We believe that detection of KRAS/BRAF mutation only in the primary colorectal tumor is not adequate before cetuximab and panitumumab therapies. Expanded studies with prospective clinical trials with a larger number of CRC cases are needed in order to establish the clinical correlation between the mutation of KRAS/BRAF in serum and the efficacy of anti-EGFR-based chemotherapies.

\section{Acknowledgements}

This study was supported by a key research grant (no. 05200178) from the GuangDong nature science fund, China. We thank Dr GuoPing Shen, Research Department, Cancer Center, Sun Yat-sen University, for assistance with statistical analysis. We thank Dr GuoLiang Huang, Research Department, Cancer Center, Sun Yat-sen University, for assistance with the design of the primers.

\section{References}

1. Jemal A, Siegel R, Ward E, Hao Y, Xu J and Thun MJ: Cancer statistics, 2009. CA Cancer J Clin 59: 225-249, 2009.

2. Van Cutsem E, Nordlinger B, Adam R, Köhne CH, et al: Towards a pan-European consensus on the treatment of patients with colorectal liver metastases. Eur J Cancer 42: 2212-2221, 2006.

3. Yoo PS, Lopez-Soler RI, Longo WE and Cha CH: Liver resection for metastatic colorectal cancer in the age of neoadjuvant chemotherapy and bevacizumab. Clin Colorectal Cancer 6: 202-207, 2006.

4. Andre T, Boni C, Mounedji-Boudiaf L, et al: Oxaliplatin, fluorouracil, and leucovorin as adjuvant treatment for colon cancer. N Engl J Med 350: 2343-2351, 2004.
5. Sargent DJ, Wieand HS, Haller DG, et al: Disease-free survival versus overall survival as a primary end point for adjuvant colon cancer studies: individual patient data from 20,898 patients on 18 randomized trials. J Clin Oncol 23: 8664-8670, 2005.

6. Cunningham D, Humblet Y, Siena S, et al: Cetuximab monotherapy and cetuximab plus irinotecan in irinotecan-refractory metastatic colorectal cancer. N Engl J Med 351: 337-345, 2004.

7. Van Cutsem E, Kohne CH, Hitre E, et al: Cetuximab and chemotherapy as initial treatment for metastatic colorectal cancer. N Engl J Med 360: 1408-1417, 2009.

8. Bokemeyer C, Bondarenko I, Makhson A, et al: Fluorouracil, leucovorin, and oxaliplatin with and without cetuximab in the first-line treatment of metastatic colorectal cancer. J Clin Oncol 27: 663-671, 2009.

9. Karapetis CS, Khambata-Ford S, Jonker DJ, et al: K-ras mutations and benefit from cetuximab in advanced colorectal cancer. N Engl J Med 359: 1757-1765, 2008.

10. Lièvre A, Bachet JB, Le Corre D, et al: KRAS mutation status is predictive of response to cetuximab therapy in colorectal cancer. Cancer Res 66: 3992-3995, 2006.

11. Lièvre A, Bachet JB, Boige V, et al: KRAS mutations as an independent prognostic factor in patients with advanced colorectal cancer treated with cetuximab. J Clin Oncol 26: 374-379, 2008.

12. Moroni M, Veronese S, Benvenuti S, et al: Gene copy number for epidermal growth factor receptor (EGFR) and clinical response to antiEGFR treatment in colorectal cancer: a cohort study. Lancet Oncol 6: 279-286, 2005.

13. Di Fiore F, Blanchard F, Charbonnier F, et al: Clinical relevance of KRAS mutation detection in metastatic colorectal cancer treated by Cetuximab plus chemotherapy. Br J Cancer 96: 1166-1169, 2007.

14. De Roock W, Piessevaux H, De Schutter J, et al: KRAS wild-type state predicts survival and is associated to early radiological response in metastatic colorectal cancer treated with cetuximab. Ann Oncol 19: 508-515, 2008.

15. Jhawer M, Goel S, Wilson AJ, et al: PIK3CA mutation/PTEN expression status predicts response of colon cancer cells to the epidermal growth factor receptor inhibitor cetuximab. Cancer Res 68: 1953-1961, 2008.

16. Scartozzi M,Bearzi I,Pierantoni C, et al: Nuclear factor-kB tumor expression predicts response and survival in irinotecan-refractory metastatic colorectal cancer treated with cetuximab-irinotecan therapy. J Clin Oncol 25: 3930-3935, 2007.

17. Perrone F, Lampis A, Orsenigo M, et al: PI3KCA/PTEN deregulation contributes to impaired responses to cetuximab in metastatic colorectal cancer patients. Ann Oncol 20: 84-90, 2009.

18. Loupakis F, Ruzzo A, Cremolini C, et al: KRAS codon 61 , 146 and BRAF mutations predict resistance to cetuximab plus irinotecan in KRAS codon 12 and 13 wild-type metastatic colorectal cancer. Br J Cancer 101: 715-721, 2009.

19. Amado RG, Wolf M, Peeters M, et al: Wild-type KRAS is required for panitumumab efficacy in patients with metastatic colorectal cancer. J Clin Oncol 26: 1626-1634, 2008.

20. Di Fiore F, Charbonnier F, Lefebure B, et al: Clinical interest of KRAS mutation detection in blood for anti-EGFR therapies in metastatic colorectal cancer. Br J Cancer 99: 551-552, 2008.

21. Hoshino R, Chatani Y, Yamori T, et al: Constitutive activation of the 41-/43-kDa mitogen-activated protein kinase signaling pathway in human tumors. Oncogene 18: 813-822, 1999.

22. Oliveira C, Velho S, Moutinho C, et al: KRAS and BRAF oncogenic mutations in MSS colorectal carcinoma progression. Oncogene 26: 158-163, 2007.

23. Yuen ST, Davies H, Chan TL, et al: Similarity of the phenotypic patterns associated with BRAF and KRAS mutations in colorectal neoplasia. Cancer Res 62: 6451-6455, 2002.

24. Artale S, Sartore-Bianchi A, Veronese SM, et al: Mutations of KRAS and BRAF in primary and matched metastatic sites of colorectal cancer. J Clin Oncol 26: 4217-4219, 2008.

25. Rajagopalan H, Bardelli A, Lengauer C, et al: Tumorigenesis: RAF/RAS oncogenes and mismatch-repair status. Nature 418: 934, 2002.

26. Souglakos J, Philips J, Wang R, et al: Prognostic and predictive value of common mutations for treatment response and survival in patients with metastatic colorectal cancer. Br J Cancer 101: 465-472, 2009

27. Nash GM, Gimbel M, Cohen AM, et al: KRAS mutation and microsatellite instability: two genetic markers of early tumor development that influence the prognosis of colorectal cancer. Ann Surg Oncol 17: 416-424, 2010. 
28. Lecomte T, Berger A, Zinzindohoué F, et al: Detection of freecirculating tumor-associated DNA in plasma of colorectal cancer patients and its association with prognosis. Int J Cancer 100 $542-548,2002$.

29. Pathak AK, Bhutani M, Kumar S, et al: Circulating cell-free DNA in plasma/serum of lung cancer patients as a potential screening and prognostic tool. Clin Chem 52: 1833-1842, 2006.

30. Tórtola S, Steinert R, Hantschick M, et al: Discordance between K-ras mutations in bone marrow micrometastases and the primary tumor in colorectal cancer. J Clin Oncol 19: 2837-2843, 2001

31. Albanese I, Scibetta AG, Migliavacca M, et al: Heterogeneity within and between primary colorectal carcinomas and matched metastases as revealed by analysis of Ki-ras and p53 mutations. Biochem Biophys Res Commun 325: 784-791, 2004.

32. Bai H, Mao L, Wang HS, et al: Epidermal growth factor receptor mutations in plasma DNA samples predict tumor response in Chinese patients with stages IIIB to IV non-small-cell lung cancer. J Clin Oncol 27: 2653-2659, 2009.
33. Gow CH, Chang YL, Hsu YC, et al: Comparison of epidermal growth factor receptor mutations between primary and corresponding metastatic tumors in tyrosine kinase inhibitor-naive non-small-cell lung cancer. Ann Oncol 20: 696-702, 2009.

34. Kalikaki A, Koutsopoulos A, Trypaki M, et al: Comparison of EGFR and K-RAS gene status between primary tumours and corresponding metastases in NSCLC. Br J Cancer 99: 923-929, 2008.

35. Ryan BM, Lefort F, McManus R, et al: A prospective study of circulating mutant KRAS2 in the serum of patients with colorectal neoplasia: strong prognostic indicator in postoperative follow up. Gut 52: 101-108, 2003.

36. Yen LC, Yeh YS, Chen CW, et al: Detection of KRAS oncogene in peripheral blood as a predictor of the response to cetuximab plus chemotherapy in patients with metastatic colorectal cancer. Clin Cancer Res 15: 4508-4513, 2009. 